Cell Research (2001); 11(4):253-264

http://www.cell-research.com

\title{
Molecular signal transduction in vascular cell apoptosis
}

\author{
GENG YONG JIAN
}

Center for Cardiovascular Biology and Atherosclerosis Research, Division of Cardiology, Department of Internal Medicine, University of Texas Houston Health Science Center Medical School, 6431 Fannin Street, MSB 6.045, Houston, TX 77030, USA

\section{ABSTRACT}

Apoptosis is a form of genetically programmed cell death, which plays a key role in regulation of cellularity in a variety of tissue and cell types including the cardiovascular tissues. Under both physiological and pathophysiological conditions, various biophysiological and biochemical factors, including mechanical forces, reactive oxygen and nitrogen species, cytokines, growth factors, oxidized lipoproteins, etc., may influence apoptosis of vascular cells. The Fas/Fas ligand/caspase death-signaling pathway, Bcl-2 protein family/mitochondria, the tumor suppressive gene p53, and the proto-oncogene c-myc may be activated in atherosclerotic lesions, and mediates vascular apoptosis during the development of atherosclerosis. Abnormal expression and dysfunction of these apoptosis-regulating genes may attenuate or accelerate vascular cell apoptosis and affect the integrity and stability of atherosclerotic plaques. Clarification of the molecular mechanism that regulates apoptosis may help design a new strategy for treatment of atherosclerosis and its major complication, the acute vascular syndromes.

Key words: Apoptosis, vascular cells, caspases, atherosclerosis, cytokines.

\section{INTRODUCTION}

Apoptosis represents a model of genetically programmed cell death and a major mechanism by which tissue removes unwanted, aged or damaged cells. Although cells of mammalian tissues consist of a broad diversity of phenotypes and genotypes, during the development of apoptosis, all cell types undergo similar morphological alterations include chromatin compaction and margination, nuclear condensation and fragmentation, and cell body shrinkage and blebbing[1]. Characteristic apoptotic morphology reflects a drastic self-destruction of cytoskeleton and a catabolism of intracellular macromolecules. Complex interactions between extracellular microenvironmental factors and internal gene expression oc-

\footnotetext{
* Corresponding author: Yong-Jian Geng, M.D., Ph.D.

Phone: 713-500-6607 Fax: 713-500-0658

Email: yong-jian.geng@uth.tmc.edu
}

cur prior to the initiation of apoptosis. Once activated, the apoptotic process can progress in the absence of extracellular insults. This suicidal feature of apoptosis has been well documented during embryonic development and morphogenesis as well as adult tissue turnover. Another important feature of apoptosis is that apoptotic cells or bodies usually retain an intact cellular membrane and are removed promptly by tissue macrophages or adjacent cells, thus exerting no harm to the tissue and triggering no inflammatory reaction. Scientists have been debating on the terminology and definition of apoptosis since this Greek word was first introduced by Kerr, et al[1] in 1972 for describing spontaneous cell death in malignant hepatic tissue. Indeed, many forms of cell death previously called necrosis are now regarded as apoptosis[2]. In order to define apoptosis and avoid confusion, Majno and Joris, et al have recently proposed a new term (oncosis) as an alterative of 
necrosis[2]. Thus far, there have been no universal standard methods for the diagnosis of apoptosis. The criteria of apoptosis vary from one cell type to others. Nevertheless, investigators have reached a consensus that apoptosis is a distinct form of cell death which exhibits many different features from necrotic cell death or oncosis (Tab 1).

Using a microscope just invented, a German pathologist, R. Virchow, recognized cell death in atherosclerotic lesions almost 150 years ago[3]. He described the atherosclerotic plaques as a transitional lesion turning proliferating cells into death. In 1995, several research laboratories, including ours, reported on apoptosis of vascular smooth muscle cells (SMC), endothelial cells (EC), and inflammatory cells in advanced human atherosclerotic lesions or atheroma[4-6]. Investigations on isolated plaque cells [7], [8], and experimental atherosclerosis[9],[10] also show the involvement of apoptosis in cell turnover during atherogenesis. Since then, a wealth of information has emerged with regard to the molecular mechanisms and biological impacts of vascular cell apoptosis in atherosclerosis. The goals of this review are to sum up recent advances in the studies of apoptosis in atherosclerosis and to delineate the molecular mechanisms for vascular cell apoptosis in different atherosclerotic lesions.

\section{Extracellular inducers of apoptosis in ath- erosclerosis}

Vascular cells, in particular SMC and EC, are exposed to a broad variety of biologically active environmental factors such as mechanical force, oxidative stress, radiation, heat, free radicals, lipids (cholesterol and its oxides), virus and bacterial products, and inflammatory cytokines produced by activated immune cells. Induction of apoptosis has been reported in vascular cells treated by some of the extracellular environmental and immunological factors (Tab 2).

Atherosclerotic lesions contain chemically modified lipoproteins, in particular oxidized low-density

Tab 1. Comparison in cell biology and biochemistry between apoptosis and oncosis (necrosis)

\begin{tabular}{lll}
\hline & Apoptosis & Oncosis \\
\hline Triggers & External/Internal & External \\
Dying cells & Individual & Group \\
Death process & Programmed & Accidental \\
Cell body & Shrinking & Swelling \\
Membrane & Intact & Broken \\
Lysosome & Intact & Leaking \\
Cytoskeleton & Collapse before death & Collapse after death \\
Mitochondria & Intact with Cyt-c release and ATP synthesis & Broken without ATP synthesis \\
Nucleus & Shrinking and fragmented & Enlarged and broken \\
Chromatin & Condensation & Clumping \\
DNA & Internucleosomal fragmentation & Randomized fragmentation \\
Caspases & Early activation & Uncertain \\
Inflammation & Rare & Common \\
\hline
\end{tabular}

Tab 2. Inducers and inhibitors of apoptosis in atherosclerosis

\begin{tabular}{ll}
\hline Inducers & Inhibitors \\
\hline OxLDL & Shear stress \\
Oxysterols & NO at low levels \\
Reactive oxygen species & VEGF \\
Reactive nitrogen species (NO at high levels) & bFGF \\
$\mathrm{X}-, \quad \gamma$, and UV radiation & Cowpox virus CremA \\
Heat & Baculovirus protein p35 \\
Cytokines & The IAP protein family \\
Fas ligand & Microorganism \\
\hline
\end{tabular}


lipoprotein (oxLDL), which has been considered as a major cytotoxic component in atherosclerosis. Cholesterol and its esters per se have little pro-apoptotic effects but become cytotoxic after oxidation. Some of oxysterols[11] in the oxLDL particles may be partially responsible for apoptotic effects of oxLDL. Oxysterol-mediated apoptosis is important in the formation of a necrotic lipid core where concentrations of oxysterols are higher than in other regions [11]. Mechanisms by which oxLDL may trigger apoptosis have not been completely understood. Recent studies suggest that activation[12] of caspases and sphingomyelinase[13] as well as suppression of the nuclear transcription factor NF-kkB[14] may contribute to oxLDL-induced apoptosis. Although native free cholesterol is not cytotoxic, high levels of cholesterol oxides in the cell membrane can trigger foam cell death by apoptosis[15].

Atherosclerosis is an inflammatory, fibroproliferative arterial disease, characterized by intimal infiltration of many immune cells and lipidrich foam cells as well as the deposition of lipids and connective tissue[16]. Immune cells, in particular macrophages and T lymphocytes (two major cellular components in cellular immunity), are activated in the local inflammatory or, more precisely, immune responses to antigenic substances[17]. The antigenic substances may include oxidized lipoproteins and some stress proteins such as heat shock protein-60. Cells of atherosclerotic plaques can produce the proinflammatory cytokines, tumor necrosis factor (TNF) and interleukin-1 (IL-1) (two major products of ac-

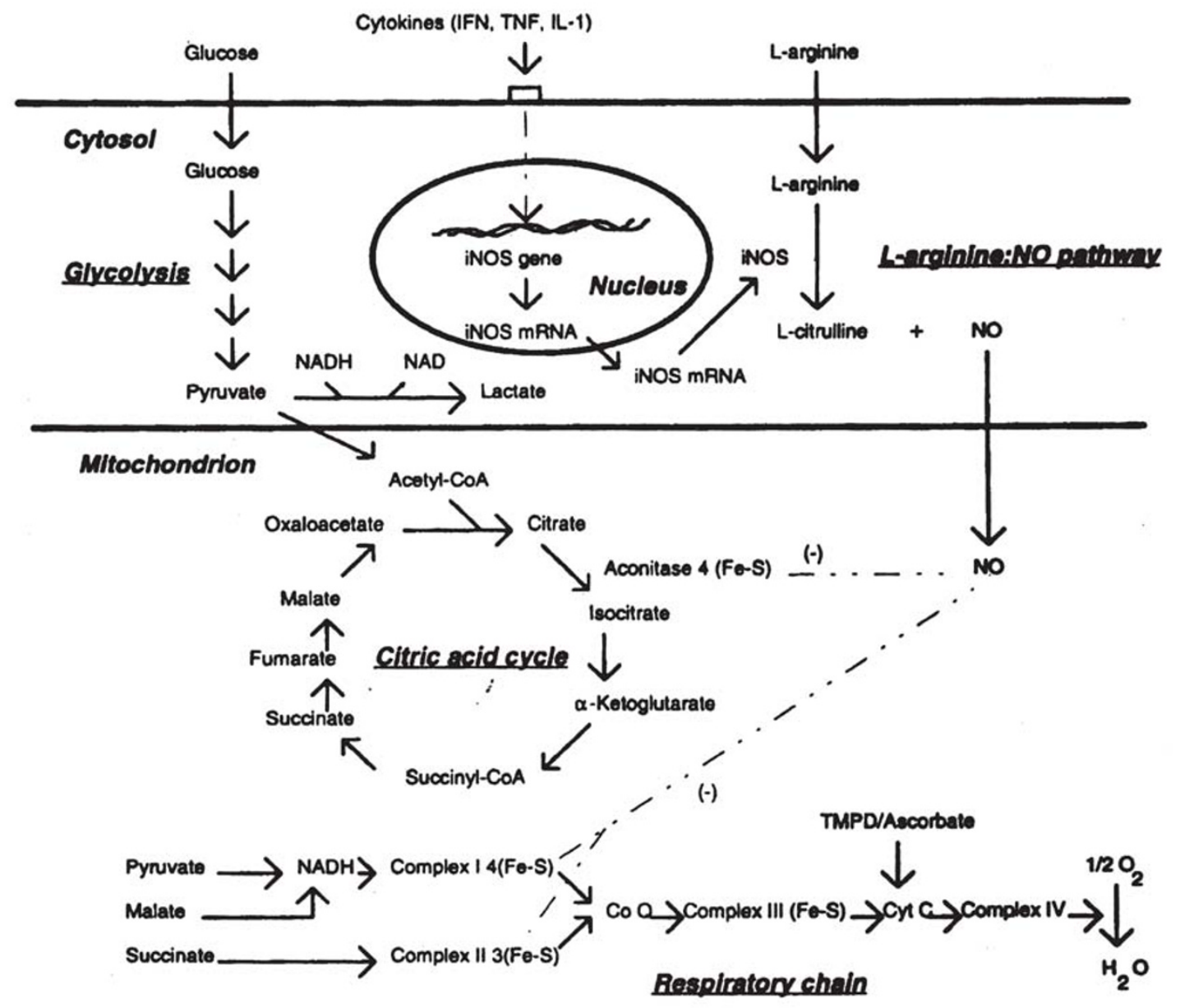

Fig 1. Schematic presentation of NO production and inhibition of mitochondrial respiration in vascular SMC exposed to proinflammatory cytokines 
tivated macrophages and, to certain extent, by vascular smooth muscle cells) and interferon- $\gamma$ (IFN-g (a cytokine of activated $\mathrm{T}$ lymphocytes)[18]. These cytokines can synergistically induce activation of the sphingomyelin-ceramide signal pathway[19] as well as the L-arginine-nitric oxide (NO) pathway[20-22]. Both the signal transduction pathways have been shown to participate in initiation and enhancement of apoptosis in vascular SMC[23],[24].

Each of the pro-inflammatory cytokines has its own biological effect on vascular cell proliferation and death. For instance, TNF and IL-1 stimulate SMC growth whereas exposure to IFN- $\gamma$ inhibits SMC proliferation. There is, however, a synergy in promoting new gene transcription between these cytokines. One of the most striking synergistic actions is induction of nitric oxide synthase, an enzyme responsible for production of $\mathrm{NO}$, a gaseous free radical with potent biological effects on contractility and metabolism of vascular SMC (Fig 1). The cytokine-induced production of NO may have a deleterious effect on SMC. At high concentrations, NO can attack several important iron-containing enzymes involved in DNA synthesis and mitochondrial respiration, leading to apoptosis of the target cells [24]. The pro-apoptotic effect of NO is markedly enhanced when NO reacts with other reactive oxygen species such as hydrogen peroxide (I) and forms the cytotoxic nitrogen reactive intermediate, peroxide nitrite. The iron-containing enzymes in mitochondria that are sensitive to NO include the complex I and complex II in the respiratory chain. Since mitochondrial dysfunction plays a critical role in the development of apoptosis (see below), the nitrosylation of iron-enzymes may not only inhibit ATP synthesis but also trigger an apoptotic cascade.

In addition to TNF, other members of the TNF superfamily also play important roles in regulation of apoptosis. The fascinating death-signaling protein, Fas/apo-1/CD95, is a surface antigen (CD95) that belongs to the TNF receptor superfamily. Surface Fas antigen ligation with anti-Fas antibodies or Fas ligand can induce apoptosis in activated T lymphocytes[25]. Many immune cells such as macrophages (the professional cell type that can present antigens) and $\mathrm{T}$ cells (the antigen-responding cells critical for a specific immune reaction) have been found to infiltrate human atherosclerotic lesions[17]. These immune cells can be activated by local and systematic factors and, thereafter, produce bioactive substances such as Fas ligand, granzymes and perforin, which can kill target cells by apoptosis.

Several lines of evidence show that the Fas/Fas ligand-caspase death pathway plays an important role in induction of apoptosis in atherosclerotic plaques[26-28]. Expression of Fas has been found in both inflammatory cells and vascular cells such as SMC and EC[26-28]. Interestingly, the vascular cells can also express the ligand for Fas which is believed to eliminate T cell infiltrate[29]. Over expression of FasL has been shown to promote atherosclerosis in a rabbit experimental atherosclerosis model[30]. Compared to its receptor, the Fas ligand expression appears to be limited in certain regions where inflammatory cells are abundant. There are increased numbers of vascular cells bearing markers for apoptosis in the areas of atherosclerotic plaques with abundant macrophages and T lymphocytes[6],[28]. Although vascular SMC express Fas, under normal conditions the cells do not undergo apoptosis even in the presence of FasL or agonistic anti-bodies[28]. The Fas expression and Fas/FasL engagement per se seem not to trigger apoptotic death in cultured human SMC. Incubation of SMC with agonistic antiFas immunoglobulin M (IgM) does not induce significant apoptosis in human aortic SMC[28]. AntiFas IgM can, however, significantly reduce viability and increase internucleosomal DNA fragmentation in SMC pretreated with the cytokines TNF, IL-1 and IFN- $\gamma$. Moreover, priming with these cytokines may enhance expression of Fas and provide additional death-promoting signals for initiation or acceleration of the apoptotic cascade in the plaque cells. Analysis of markers for apoptosis indicates that many T lymphocytes may have undergone apoptosis when they infiltrate into atherosclerotic lesions[31]. Expression of FasL in vascular cells and macrophages may partially explain why some of the plaque cells may escape apoptotic attack by activated T lymphocytes and have prolonged lifespan in the lesions.

\section{Environmental inhibitors of apoptosis in atherosclerosis}

Many inhibitors of apoptosis have been identified for the past several years including shear stress, growth factors, antioxidants and vitamins (Tab 2). 
These inhibitors counteract apoptotic effects of cytotoxic factors, maintain integrity of endothelium, and increase cellularity of the vessel wall.

Mechanical force generated by blood flow can induce remodeling of the endothelial layer as well as the vessel wall[32]. Lack of shear stress may induce apoptosis of EC[33] and, by contrast, application of shear stress can inhibit apoptosis of human EC[34]. The inhibitory effect of shear stress on apoptosis of EC may be associated with production of low levels of NO through activation of endothelial NO synthase. In contrast to cytokine- induced NO synthase, constitutive endothelial NOS generates small amounts of NO that may inhibit caspase activities and become protective to endothelial cell apoptosis[35]. Analysis of apoptosis in human atherosclerotic lesions shows a systematic preferential occurrence of apoptosis in the downstream parts of plaques where low-flow and low-shear stress prevail[36].

Some growth factors may also protect cells from apoptosis. For instance, vascular endothelial cell growth factor (VEGF) can inhibit apoptosis of bone marrow cells induced by radiation[37]. Basic fibroblast growth factor (bFGF) and its antisense are reported to inhibit apoptosis of EC induced by $\mathrm{TNF} \alpha$. The antisense therapy also triggers apoptosis of vascular SMC[38]. This growth factor also prevents apoptosis induced by serum starvation[39-41]. Protein kinase-C has been reported to mediate the protective effect of fibroblast growth factor[39],[40].

Because oxidative stress can injure vascular cells and promote apoptosis as stated above, Anti-oxidant therapy has been proposed[42]. Evidence has emerged that antioxidants may have inhibitory effects on cell apoptosis. For instance, during 7ketocholesterol-induced apoptosis of U937 cells, a rapid decrease occurs in the cellular glutathione content, the oxidation of polyunsaturated fatty acids, and the production of reactive oxygen species. Administration of glutathione can prevent the 7ketocholesterol-induced apoptotic cell death[42].

\section{Intracellular apoptosis machinery in ath- erosclerosis}

In the $1990 \mathrm{~s}$, many gene products have been identified to serve as a signal transducer, a regulator, an enhancer, or an executor of apoptosis. A great diversity of the genetic background, cell structure, metabolism, and function exists between mammalian cells. However, there has been no universal death pathway that accounts for every cell death. Just like any other types of tissue cells, vascular cells appear to have their own apoptosis machinery ready for regulation and execution of apoptosis upon receiving death signals. Nevertheless, recent investigations have pointed to the possibility that certain enzymatic systems play central roles in vascular cell apoptosis, under both physiological and pathological conditions, as described in a series of reviews recently published [43-48]. This review focuses on three major apoptosisregulatory or executing systems located in cytoplasm, mitochondria and nuclei, respectively.

\section{Caspases}

In the cytoplasm, there is a group of aspartatespecific cysteinyl proteases or caspases[49]. Thus far, more than 14 members of the caspase family have been identified in non-cardiac tissues, particularly in the immune and nervous systems. All members of the caspase family show a similar substrate cleavage at an aspartate residue, and they are expressed as proenzymes. The caspase family participates in downstream events of death signa-ling by the members of TNF receptor superfamily (e.g., Fas and TNF receptor-1) which share a related intracellular "death domain" of about 70 amino acid residues[25]. Fasassociated protein with death domain (FADD)/ MORT1 and receptor-interacting protein (RIP) and TNFR-1-associated death domain protein (TRADD) serve as downstream factors to induce apoptosis. Based upon their functions, caspases can be divided into three groups: the first group (caspases 1, 4, 5, 11-14) with a limited role in apoptosis mainly participates in inflammation; the second group (caspase 2, 8-10) serves as initiators of apoptosis; and the third group (caspases 3, 6 and 7) act as effectors of apoptosis.

The exact picture of how activation of caspases leads to cell death remains unclear. Available evidence largely from the Fas/TNF-R death-signaling model indicates two distinct but related pathways (Fig 2). In pathway one[25], Fas-FasL ligation trimerizes the receptor, recruits cofactors such as 
FADD and RIP, and then activates caspases 8 and 2 by autoproteolysis and oligomerization. Subsequently, the effector caspases 3, 6 and 7, are also proteolytically activated, resulting in clea-vage of a variety of cellular substrates. In pathway two, many cytotoxic substances may attack mitochondria, translocate pro-apoptotic members of the Bcl-2 family to mitochondria, alter voltage-dependent channels in the mitochondrial membrane, and release cytochrome-c (cyt-c). Via the adaptor molecule, Apaf-1, cyt-c and caspase-9 form a complex, which, in turn, activates the effector caspases and triggers the caspase cascade. Interestingly, in some cells, caspase8 activation can also activate the mitochondrial death pathway by cleavage of Bid, a pro-apoptotic member of the Bcl-2 family. Although the two pathways of apoptosis can operate in the same cells, studies suggest that certain types of cells may predominantly utilize one of the mechanisms for apoptosis. In general, pathway one is more active than pathway two in the receptor-mediated cell death[25]. Conversely, pathway two is more prominent in death induced by cytotoxic agents[49],[50].

Expression of caspases has been reported in human and animal atherosclerotic plaques[6] and in cultured vascular cells[51]. However, the biological functions of caspases in atherosclerotic lesions are largely unknown. Many intracellular proteins have been shown as substrates for caspases. Among them, the proteins associated with nucleases play an important role in the final phase of the caspase cascade. Normally, cells are protected from nucleo-lysis by producing inhibitors of the nucleases. Caspase 3 can cleave I ${ }^{C A D} / \mathrm{DFF} 45$, an inhibitor of the nuclease responsible for DNA fragmentation, CAD (caspaseactivated deoxyribonuclease)[49]. In addition, activated caspase 3 also cleave gelsolin, an actin-associated protein that may have influence on the activities of DNase I. In cultured SMC, cytokine-induced activation of caspase 3 triggers an apoptosis cascade in which fragmented gelsolin depolymerizes actin, promotes collapse of cytoskeleton, and induces activation of nucleases that degrade DNA[51].

\section{Bcl-2 and mitochondria}

The Bcl-2 protein family represents another group of cellular proteins that are important Regu- lators of apoptosis in mammalian cells. Bcl-2 has been found abundantly in the mitochondrial membrane[52]. The Bcl-2 family has more than 15 members[50]. Based upon differences in regulation of apoptosis, members of this family can be divided into two subgroups. Each of them contains five or more function- and structure-related proteins. The first group is composed of the anti-apoptotic proteins such as Bcl-2, Bid, Bcl-X, Mcl-1, Bcl-w and A1; members of the second group are pro-apoptotic including Bax, Bak, Bad, Bik, Hrk, Bid and Bcl-xs. The mechanism for inhibition of apoptosis by Bcl-2 has not been completely clarified. Bcl-2 may exert an antioxidant effect on stressed cells, prevent release of mitochondrial cytochrome $\mathrm{C}$, and form complexes with other pro-apoptotic molecules such as Bax and Bak.

Rapidly accumulating literature indicates the pivotal role of mitochondria in apoptosis[52], [53]. The regulatory effects of mitochondria on apoptosis are threefold: 1) inhibition of mitochondrial respiration. As discussed above, the res-piratory chain inhibition may occur when large amounts of NO are synthesized in cytokine-stimulated vascular cells; 2) production of cytotoxic reactive oxygen species or change of cellular reduction-oxidation (redox) potential; and 3) release of pro-apoptotic molecules including cyt-c and apoptosis inducing factor (AIF). Many Bcl-2 family proteins reside in the mitochondrial outer membrane where they form a pore structure that surprisingly resembles some bacterial toxins enhancing proton extrusion. The release of cyt-c is closely associated with the mitochondrial inner transmembrane potential $\Delta \Psi \mathrm{m}$ ). When apoptotic stimuli attenuate Bcl-2 function, a rapid drop or collapse of $\Delta \Psi \mathrm{m}$ may occur, causing rapid release of cyt-c and other pro-apoptotic proteins. Cytosolic cyt-c then forms, together with Apaf-1 and caspase 9, an "apoptosome" to orchestrate activation of other caspases and the biochemical execution of apoptosis.

\section{p53 and oncogenes 7}

Nuclear proteins, in particular those related to oncogenes or anti-oncogenes[54], such as p53 and c-myc, also play important roles in regulation of apoptosis of vascular cells during atherogenesis. The tumor suppressive gene p53 functions as an antioncogene associated with up-regulation of apoptosis 


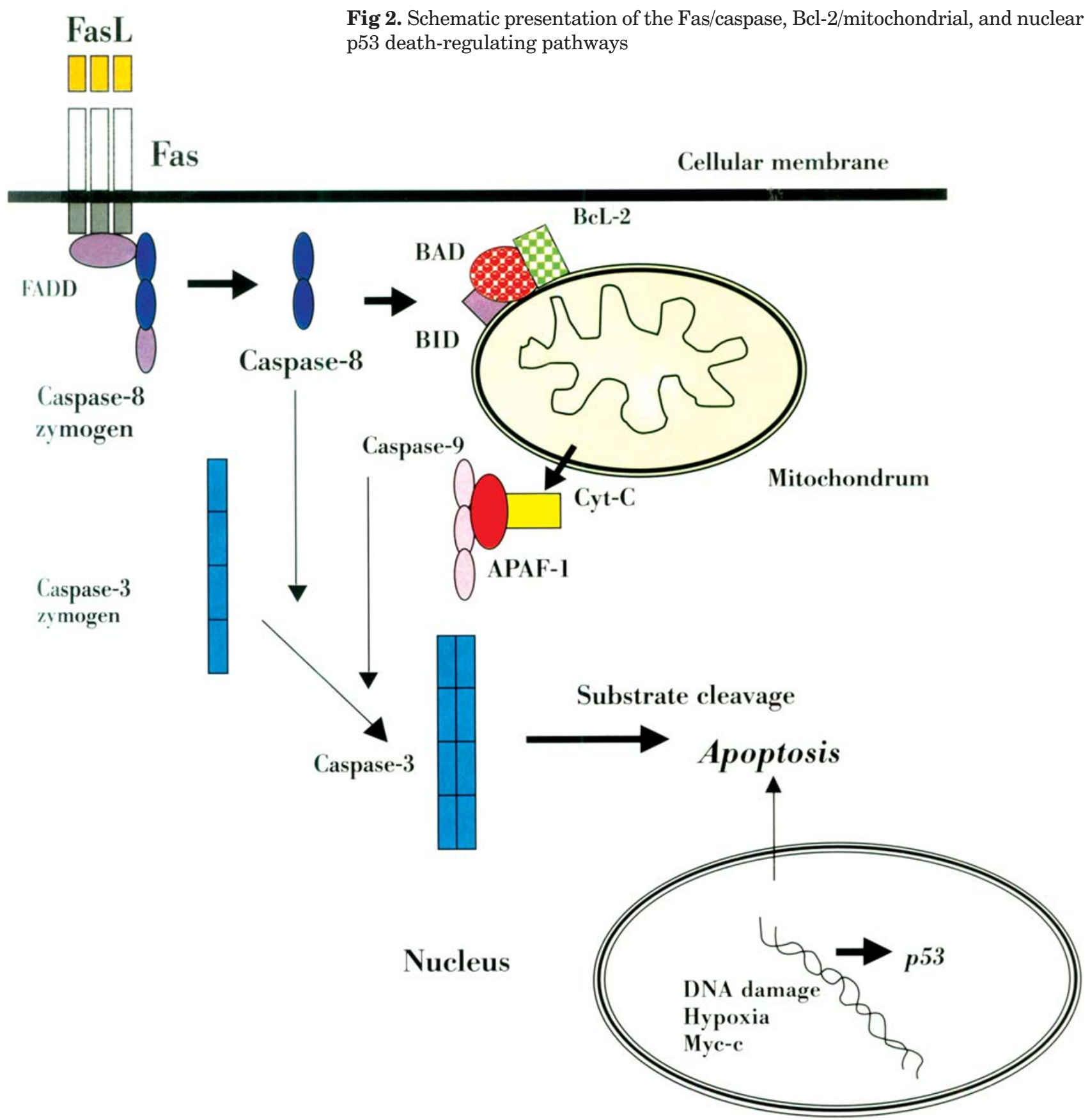

(Fig 2). Wild-type p53 arrests cell proliferation and may hold cells with DNA damage in G1 phase. During DNA repairing, some p53-expressing cells oppose the G1 block and enter the suicide pathway. Mutation or interaction with viral products such as SV 40T antigen and the products of human cytomegalovirus can cause dysfunction of p53 and, in turn, inhibit apoptosis. A high frequency of p53 mutation has been reported to exist prominently in certain cancer cells. Cells in normal blood vessels express little p53, but immunoreactive p53 was reported to exist in vascular cells undergoing apoptosis[4].In vitro studies also show evidence that $\mathrm{p} 53$ regulates apoptosis in cultured SMC[7],[8]. Cells in the plaques but not in normal arterial tissue can express wild types of p53. Using an in vitro system, Bennett et al 
[7],[8] reported that p53 mediates induction of apoptosis by deprivation of growth factors and transfection with c-myc in vascular SMC.

Different oncogenes may interact in terms of controlling apoptosis. Compared with normal cells, SMC from atherosclerotic plaques show a higher ratio of the active (hypophosphorylated) to the inactive (phosphorylated) form of the tumor suppressor gene $\mathrm{Rb}$ (retinoblastoma gene product) and a lower level of E2F transcriptional activity[55]. Suppression of $\mathrm{RB}$ alone increases rates of cell proliferation and apoptosis and inhibited cell senescence in normal VSMCs. Suppression of p53 and RB together exerts similar effects. In contrast, inhibition of RB binding to E2F or ectopic expression of E2F-1 in plaque cells induces massive apoptosis, which requires suppression of p53 to rescue cells. Suppression of RB and p53 together increases cell proliferation and delays senescence but fails to immortalize plaque cells. Inhibition of p53 alone has minimal effects on plaque cells but increases the lifespan of normal SMC. After balloon catheterization or angioplasty, cells respond to the mechanical injury by proliferation as a wound healing process. The cells become apoptotic later on as scar tissue forms. Speir et al[30] reported that some of these cells in restenosis lesions can express wild-type p53, an inducer of apoptosis. They further observed that human cytomegaloviris interacts with the apoptosispromoter p53, leading to inactivation of this gene product and thereby a prolonged life span of the cells or hypertrophy. These observations raise the possibility that the normal process of apoptosis may be attenuated, resulting in an increased accumulation of cells in the intima, (i.e., restenosis).

The proto-oncogene c-myc mediates both cell death and proliferation in a manner that is dependent on the levels of expression. It functions as a nuclear phosphoprotein with certain properties of transcription factors[54]. Activation of c-myc usually stimulates growth of fibroblasts in regular cell cultures containing serum or growth factors. In the cultures containing little or no serum, however, cells over expressing c-my readily undergo apoptosis. Deregulation of c-myc causes apoptosis of the vascular SMC deprived of growth factors or treated with cytokines such as IFN- $\gamma$ [56]. The gene p53 has been shown to mediate the apoptotic effect of c-myc in various cell lines.
Apoptosis in progression and regression of atherosclerosis

A typical atherosclerotic lesion usually develops in the tunica intima of the arteries where cellular components, lipids, and connective tissue accumulate, yielding a fibrofatty plaque or atheroma that focally thickens the intima and subsequently narrows the arterial lumen. Counteracting proliferation, apoptosis may help remove excess numbers of cells that infiltrate and accumulate in the intimal compartment. Removal of inflammatory and lipid-laden foam cells may lead to regression of atherosclerosis.

In contrast, attenuation of apoptosis may increase the tissue cellularity and promote intimal hyperplasia. Recent studies suggest the possibility that an anti-apoptotic mechanism may result in foam cell accumulation in atherosclerotic lesions. In spite of the presence of cytotoxic substances in atherosclerotic lesions, many lipid-laden foam macrophages reside in atherosclerotic lesions, especially in the lipid core area where the cells are exposed to a very harsh microenvironment. Expression of anti-apoptotic genes may practically explain the resistance of foam cells to apoptosis. Employing an in vitro model, we have recently found that the class A scavenger receptor responsible for uptake of chemically modified lipoproteins may protect macrophages from apoptosis induced by oxysterols and oxLDL[57]. The mechanism by which this scavenger receptor inhibits apoptosis in macrophages remains to be determined. Some evidence implies that the scavenger receptorassociated resistance of foam cells to apoptosis may involve signal transduction since expression of this receptor inhibits apoptosis induced by sodium fluoride, a global activator of GTP-binding proteins [58].

Although apoptosis may prevent cell accumulation in atherosclerosis, recent pathological investigations, however, have collected data suggesting the involvement of apoptosis in the development and evolution of advanced atherosclerotic lesions or atheroma[43]. The center of atherosclerotic plaques contains, in addition to lipids and connective tissue, many dead cells or cell debris, thus referred to as a necrotic lipid core. Massive apoptosis may occur as atherosclerosis progresses, often yielding a lesion containing a dense extracellular matrix with a rela- 
tively low-density of cells. The apoptosis-mediated conversion of a hypercellular atherosclerotic lesion to the more cytopenic fibrotic atheroma is evident in advanced atherosclerosis. Analysis of cell death by in situ end-labeling of DNA fragments (TUNEL technique) and by morphological observation indicates that many cells in the atheromatous lesions bear the markers of apoptosis even though necrotic cell death may also occur in the lipid core area of atherosclerotic lesions. Thus, apoptosis may appear abnormally in atherosclerotic lesions, contributing to the formation of two major pathological changes seen often in advanced atherosclerotic plaques (i.e., the "necrotic lipid core" and hypocellular fibrotic lesion). Advanced atheroma contains many apoptotic cells as detected by in situ terminal labeling of DNA strand breaks[59]. Cells isolated from atherosclerotic arteries have been proven difficult to grow in vitro [8]. On the other hand, fewer cells show proliferating markers when stained with antibodies against the proliferating cell nuclear antigen or cyclins. These observations challenge the traditional view that thickening of the intima of atherosclerotic arteries is due to cell growth.

Many laboratories reported a high percentage of apoptotic cells in advanced, progressive atherosclerotic plaques. It appears to be a paradox because the tissue volume should decrease when the levels of apoptosis are substantially higher than that of proliferation. However, apoptosis is not always linked to reduction of tissue volume. The fact that many apoptotic cells accumulate in the plaques suggests that the system for scavenging the dead cells poorly operates in atherosclerosis. Some apoptotic cells, or bodies in the plaques, stay at a "mummified" stage rather than being removed by phagocytosis. The following mechanisms may lead to detention of apoptotic cells or bodies in atherosclerotic lesions: (1) intracellular accumulation of lipids attenuates the ability of macrophages and SMC to engulf and digest apoptotic cells; (2) phagocytosis decreases due to increased apoptosis of macrophages in the lesions; and (3) cross-linking of macromolecules such as proteins, nucleic acid, and other carbohydrates stabilizes apoptotic cells in the tissues.

\section{Apoptosis in development of acute vascu- lar syndromes}

Plaque rupture is a key event in acute vascular syndrome that can cause severe thrombotic complications and provoke unstable angina pectoris[60-62]. Vascular SMC can synthesize a variety of extracellular matrix macromolecules that stabilize the plaques. Pathological investigations show that inflammatory cell infiltration increases the risk of plaque disruption[60]. Production of apoptosis-promoting cytotoxic substances by activated inflammatory cells or immune cells may induce massive death of vascular SMC, which may in turn weaken and destabilize the plaques. Also, these cells can produce a variety of matrix metalloproteinases which, when activated, degrade the extracellular matrix leading to plaque rupture. Although apoptosis usually occurs more frequently in the intimal cells, under certain pathological conditions this type of cell death may attack medial SMC. High levels of apoptosis have been found in the medial SMC of arteries with aneurysms. In atherosclerotic aortic aneurysms, macrophages and T lymphocytes infiltrate the arterial wall and produce death-promoting proteins (perforin, Fas, and FasL)[63].

Thrombosis promptly developing on ruptured plaques represents a major event that threatens life in acute vascular syndromes. Both apoptotic EC and SMC have been reported to be highly pro-coagulative[64-66]. While no antigenic or functional tissue factor exists, rapid exposure of membrane phosphatidylserine and loss of the anticoagulant membrane components in apoptotic EC can occur, which may contribute to the pro-coagulative effect. Apoptotic vascular SMC possess a significant thrombin-generating capacity secondary to phosphatidylserine exposure. Apoptotic cells within atherosclerotic plaques may allow local thrombin activation, thereby contributing to disease progression[65]. These data also point to an important scenario that, if they are not promptly removed, apoptotic cells or bodies may become thrombogenic or pro-inflammatory.

\section{Conclusions}

Apoptosis represents a major mechanism responsible for regulation of the cellularity of the arterial wall during atherogenesis. Many environmental and endogenous factors can influence apoptosis through various signal transduction pathways or enzymatic systems. Among these factors triggering 
apoptosis are modified lipids, pro-inflammatory cytokines, and Fas ligands produced by activated immune cells. The cytokine-induced NO synthesis, Fas-mediated caspase activation, Bcl-2/mitochondrial dysfunction, and p53 antioncogene expression, play roles in initiation and progression of vascular cell apoptosis. Abnormality of apoptosis may occur in atherosclerosis, leading to a gross accumulation of intimal cells in the development of atherosclerosis. In advanced atheroma, during acute vascular syndromes in particular, massive apoptosis of vascular cells may weaken the fibrous cap, promote thrombosis, and increase the risk of plaque disruption. Further clarification of the molecular mechanisms for vascular apoptosis may help design new therapeutic strategies for treatment of atherosclerosis and acute vascular syndromes.

\section{ACKNOWLEDGMENT}

This work is supported by grants (R01HL-59249 and ROIHL-69509) from National Institutes of Health, USA.

\section{REFERENCES}

[1] Kerr J, Wyllie A and Currie A. A basic biological phenomenon with wide ranging implications in tissue kine-tics. Brit J Cancer 1972; 26:239-57.

[2] Majno G and Joris I. Apoptosis, oncosis, and necrosis. An overview of cell death. Am J Pathol 1995; 146:3-15.

[3] Virchow R. Die Cellularpathologie: Sechszehnte verlesung, 14 April 1858, George Olms, Hildesheim, Germany 1966: 317-29.

[4] Isner JM, Kearney M, Bortman S and Passeri J. Apoptosis in human atherosclerosis and restenosis. Circulation 1995; 91:2703-11.

[5] Han DK, Haudenschild CC, Hong MK, Tinkle BT, Leon MB and Liau G. Evidence for apoptosis in human atherogenesis and in a rat vascular injury model. Am J Pathol 1995; 147:267-77.

[6] Geng YJ and Libby P. Evidence for apoptosis in advanced human atheroma. Co-localization with interleukin-1 betaconverting enzyme. Am J Pathol 1995; 147:251-66.

[7] Bennett MR, Evan GI and Schwartz SM. Apoptosis of rat vascular smooth muscle cells is regulated by p53-dependent and -independent pathways. Circ Res 1995; 77:26673.

[8] Bennett MR, Evan GI and Schwartz SM. Apoptosis of human vascular smooth muscle cells derived from normal vessels and coronary atherosclerotic plaques. J Clin Invest 1995; 95:2266-74.

[9] Kockx MM, De MG, Muhring J, Bult H, Bultinck J and Herman AG. Distribution of cell replication and apoptosis in atherosclerotic plaques of cholesterol-fed rabbits. Atherosclerosis 1996; 120:115-24.
[10] Bochaton-Piallat ML, Gabbiani F, Redard M, Desmouliere A and Gabbiani G. Apoptosis participates in cellularity regulation during rat aortic intimal thickening. Am J Pathol 1995; 146:1059-64.

[11] Thompson EB and Ayala-Torres S. Oxysterols and apoptosis: evidence for gene regulation outside the cholesterol pathway. Crit Rev Biochem Mol Biol 1999; 34: 25-32.

[12] Harada-Shiba M, Kinoshita M, Kamido H and Shimokado $\mathrm{K}$. Oxidized low density lipoprotein induces apoptosis in cultured human umbilical vein endothelial cells by common and unique mechanisms. J Biol Chem 1998; 273: 9681-7.

[13] Escargueil-Blanc I, Andrieu-Abadie N, Caspar-Bauguil S, Brossmer R, Levade T, Negre-Salvayre A and Salvayre R. Apoptosis and activation of the sphingomyelin-ceramide pathway induced by oxidized low density lipoproteins are not causally related in ECV-304 endothelial cells. J Biol Chem 1998; 273:27389-95.

[14] Heermeier K, Leicht W, Palmetshofer A, Ullrich M, Wanner C and Galle J. Oxidized LDL Suppresses NF-kB and Overcomes Protection from Apoptosis in Activated Endothelial Cells. J Am Soc Nephrol 2001; 12:456-63.

[15] Kellner-Weibel G, Geng YJ and Rothblat GH. Cytotoxic cholesterol is generated by the hydrolysis of cytoplasmic cholesteryl ester and transported to the plasma membrane. Atherosclerosis 1999; 146:309-19.

[16] Ross R. Atherosclerosis-an inflammatory disease. N Engl J Med 1999; 340:115-26.

[17] Hansson GK. Cell-mediated immunity in atherosclerosis. Curr Opin Lipidol 1997; 8:301-11.

[18] Hansson GK, Stemme S, Geng YJ and Holm J. Can immunocompetent cells and their cytokines play a role in atherogenesis? Nouv Rev Fr Hematol 1992; 34:S43-6.

[19] Auge N, Negre-Salvayre A, Salvayre R and Levade T. Sphingomyelin metabolites in vascular cell signaling and atherogenesis. Prog Lipid Res 2000; 39:207-29.

[20] Geng YJ, Almqvist M and Hansson GK. cDNA cloning and expression of inducible nitric oxide synthase from rat vascular smooth muscle cells. Biochim Biophys Acta 1994; 1218:421-4.

[21] Geng YJ, Petersson AS, Wennmalm A and Hansson GK. Cytokine-induced expression of nitric oxide synthase results in nitrosylation of heme and nonheme iron proteins in vascular smooth muscle cells. Exp Cell Res 1994; 214:418-28.

[22] Geng YJ, Hansson GK and Holme E. Interferon-gamma and tumor necrosis factor synergize to induce nitric oxide production and inhibit mitochondrial respiration in vascular smooth muscle cells. Circ Res 1992; 71:1268-76.

[23] Chatterjee S. Sphingolipids in atherosclerosis and vascular biology. Arterioscler Thromb Vasc Biol 1998; 18:152333.

[24] Geng YJ, Hellstrand K, Wennmalm A and Hansson GK. Apoptotic death of human leukemic cells induced by vascular cells expressing nitric oxide synthase in response to gamma- interferon and tumor necrosis factor-alpha. Cancer Res 1996; 56:866-74.

[25] Ashkenazi A and Dixit VM. Death receptors: signaling and 
modulation. Science 1998; 281:1305-8. This review sums up research on Fas-initiated, caspase-mediated apoptosis.

[26] Cai W, Devaux B, Schaper W and Schaper J. The role of Fas/ APO 1 and apoptosis in the development of human atherosclerotic lesions. Atherosclerosis 1997; 131:177-86.

[27] Fukuo K, Nakahashi T, Nomura S, Hata S, Suhara T, Shimizu M, Tamatani M, Morimoto S, Kitamura Y and Ogihara T. Possible participation of Fas-mediated apoptosis in the mechanism of atherosclerosis. Gerontology 1997; 1:35-42.

[28] Geng YJ, Henderson LE, Levesque EB, Muszynski M and Libby P. Fas is expressed in human atherosclerotic intima and promotes apoptosis of cytokine-primed human vascular smooth muscle cells. Arterioscler Thromb Vasc Biol 1997; 17:2200-8.

[29] Walsh K and Sata M. Is extravasation a Fas-regulated process? Mol Med Today 1999; 5:61-7.

[30] Schneider DB, Vassalli G, Wen S, Driscoll RM, Sassani AB, DeYoung MB, Linnemann R, Virmani R and Dichek DA. Expression of Fas ligand in arteries of hypercholesterolemic rabbits accelerates atherosclerotic lesion formation. Arterioscler Thromb Vasc Biol 2000; 20:298308.

[31] Bjorkerud S and Bjorkerud B. Apoptosis is abundant in human atherosclerotic lesions, especially in inflammatory cells (macrophages and T cells), and may contribute to the accumulation of gruel and plaque instability. Am J Pathol 1996; 149:367-80.

[32] Langille BL. Arterial remodeling: relation to hemodynamics. Can J Physiol Pharmacol 1996; 74:83441.

[33] Kaiser D, Freyberg MA and Friedl P. Lack of hemodynamic forces triggers apoptosis in vascular endothelial cells. Biochem Biophys Res Commun 1997; 231:586-90.

[34] Dimmeler S, Haendeler J, Rippmann V, Nehls M and Zeiher AM. Shear stress inhibits apoptosis of human endothelial cells. Febs Lett 1996; 399:71-4.

[35] Dimmeler S, Haendeler J, Nehls M and Zeiher AM. Suppression of apoptosis by nitric oxide via inhibition of interleukin-1beta-converting enzyme (ICE)-like and cysteine protease protein (CPP)-32-like proteases. J Exp Med 1997; 185:601-7.

[36] Tricot O, Mallat Z, Heymes C, Belmin J, Leseche G and Tedgui A. Relation between endothelial cell apoptosis and blood flow direction in human atherosclerotic plaques. Circulation 2000; 101:2450-3.

[37] Katoh O, Tauchi H, Kawaishi K, Kimura A and Satow Y. Expression of the vascular endothelial growth factor (VEGF) effect of VEGF on apoptotic cell death caused by ionizing radiation. Cancer Res 1995; 55:5687-92.

[38] Fox JC and Shanley JR. Antisense inhibition of basic fibroblast growth factor induces apoptosis in vascular smooth muscle cells. J Biol Chem 1996; 271:12578-84.

[39] Araki S, Simada Y, Kaji K and Hayashi H. Role of protein kinase $\mathrm{C}$ in the inhibition by fibroblast growth factor of apoptosis in serum-depleted endothelial cells. Biochem Biophys Res Commun 1990; 172:1081-5.

[40] Haimovitz FA, Balaban N, McLoughlin M, Ehleiter D, Michaeli J, Vlodavsky I and Fuks Z. Protein kinase C mediates basic fibroblast growth factor protection of endothelial cells against radiation-induced apoptosis. Cancer Res 1994; 54:2591-7.

[41] Kondo S, Yin D, Aoki T, Takahashi JA, Morimura T and Takeuchi J. bcl-2 gene prevents apoptosis of basic fibroblast growth factor- deprived murine aortic endothelial cells. Exp Cell Res 1994; 213:428-32.

[42] Lizard G, Gueldry S, Sordet O, Monier S, Athias A, Miguet C, Bessede G, Lemaire S, Solary E and Gambert P. Glutathione is implied in the control of 7-ketocholesterolinduced apoptosis, which is associated with radical oxygen species production. Faseb J 1998; 12:1651-63.

[43] Geng YJ. Regulation of programmed cell death or apoptosis in atherosclerosis. Heart Vessels 1997; Suppl:76-80.

[44] Kockx MM and Herman AG. Apoptosis in atherosclerosis: beneficial or detrimental? Cardiovasc Res 2000; 45:736-46.

[45] Bennett MR. Apoptosis of vascular smooth muscle cells in vascular remodeling and atherosclerotic plaque rupture. Cardiovasc Res 1999; 41:361-8.

[46] Best PJ, Hasdai D, Sangiorgi G, Schwartz RS, Holmes DR, Simari RD and Lerman A. Apoptosis. Basic concepts and implications in coronary artery disease. Arterioscler Thromb Vasc Biol 1999; 19:14-22.

[47] Walsh K and Isner JM. Apoptosis in inflammatoryfibroproliferative disorders of the vessel wall. Cardiovasc Res 2000; 45:756-65.

[48] Mallat Z and Tedgui A. Apoptosis in the vasculature: mechanisms and functional importance. Br J Pharmacol 2000; 130:947-62.

[49] Thornberry NA and Lazebnik Y. Caspases: enemies within. Science 1998; 281:1312-6.

[50] Adams JM and Cory S. The Bcl-2 protein family: arbiters of cell survival. Science 1998; 281:1322-6.

[51] Geng YJ, Azuma T, Tang JX, Hartwig JH, Muszynski M, Wu Q, Libby P and Kwiatkowski DJ. Caspase-3-induced gelsolin fragmentation contributes to actin cytoskeletal collapse, nucleolysis, and apoptosis of vascular smooth muscle cells exposed to proinflammatory cytokines. Eur J Cell Biol 1998; 77:294-302.

[52] Green DR and Reed JC. Mitochondria and apoptosis. Science 1998; 281:1309-12.

[53] Soengas MS, Alarcon RM, Yoshida H, Giaccia AJ, Hakem R, Mak TW and Lowe SW. Apaf-1 and caspase-9 in p53dependent apoptosis and tumor inhibition. Science 1999; 284:156-9.

[54] Evan G and Littlewood T. A matter of life and cell death. Science 1998; 281:1317-22.

[55] Bennett MR, Macdonald K, Chan SW, Boyle JJ and Weissberg PL. Cooperative interactions between RB and p53 regulate cell proliferation, cell senescence, and apoptosis in human vascular smooth muscle cells from atherosclerotic plaques. Circ Res 1998; 82:704-12.

[56] Bennett MR, Evan GI and Newby AC. Deregulated expression of the c-myc oncogene abolishes inhibition of proliferation of rat vascular smooth muscle cells by serum reduction, interferon-gamma, heparin, and cyclic nucleotide analogues and induces apoptosis. Circ Res 1994; 74:525-36.

[57] Liao HS, Kodama T and Geng YJ. Expression of class A scavenger receptor inhibits apoptosis of macrophages 
triggered by oxidized low density lipoprotein and oxysterol. Arterioscler Thromb Vasc Biol 2000; 20:196875.

[58] Liao HS, Matsumoto A, Itakura H, Pittman T, Kodama $\mathrm{T}$ and Geng YJ. De novo expression of the class-A macrophage scavenger receptor conferring resistance to apoptosis in differentiated human THP-1 monocytic cells. Cell Death Differ 1999; 6:245-55.

[59] Geng YJ and Libby P. Evidence for apoptosis in advanced human atheroma. Colocalization with interleukin-1 betaconverting enzyme. Am J Pathol 1995; 147:251-66.

[60] Libby P, Geng YJ, Aikawa M, Schoenbeck U, Mach F, Clinton SK, Sukhova GK and Lee RT. Macrophages and atherosclerotic plaque stability. Curr Opin Lipidol 1996; 7:330-5.

[61] Libby P, Geng YJ, Sukhova GK, Simon DI and Lee RT. Molecular determinants of atherosclerotic plaque vulnerability. Ann N Y Acad Sci 1997; 811:134-42; discussion 142-5.
[62] Shah PK. Plaque Disruption and Thrombosis: Potential Role of Inflammation and Infection. Cardiol Rev 2000; 8: 31-9.

[63] Henderson EL, Geng YJ, Sukhova GK, Whittemore AD, Knox $\mathrm{J}$ and Libby P. Death of smooth muscle cells and expression of mediators of apoptosis by T lymphocytes in human abdominal aortic aneurysms. Circulation 1999; 99:96-104.

[64] Bombeli T, Karsan A, Tait JF and Harlan JM. Apoptotic vascular endothelial cells become procoagulant. Blood 1997; 89:2429-42.

[65] Flynn PD, Byrne CD, Baglin TP, Weissberg PL and Bennett MR. Thrombin generation by apoptotic vascular smooth muscle cells. Blood 1997; 89:4378-84.

[66] Greeno EW, Bach RR and Moldow CF. Apoptosis is associated with increased cell surface tissue factor procoagulant activity. Lab Invest 1996; 75:281-9. 